\title{
A System for Improving the Management of the Lesion and Test Images of Patients
}

\author{
Sung-Bum Cho ${ }^{1}$, Kyung-Won Jin ${ }^{1}$, Jong-Soon Kim ${ }^{1}$, Soo-Jin Huh ${ }^{1,2}$, Segyeong Joo ${ }^{1,2}$ \\ ${ }^{1}$ Department of Biomedical Engineering, Asan Medical Center, Seoul, Korea \\ ${ }^{2}$ Department of Biomedical Engineering, University of Ulsan College of Medicine, \\ Seoul, Korea \\ Email:sgjoo@amc.seoul.kr \\ Received May 2013
}

\begin{abstract}
Nowadays, almost every hospital utilizes picture archiving and communications system (PACS) and electronic medical record (EMR) system to manage patient's data digitally. However, patient's pictures taken by nurses or physicians to monitor lesions or various test results are not managed well in general. Although for some disease, these pictures provide meaningful information in diagnosis and treatment. In this paper, we propose a management system for these images to improve the accessibility and storability. To manage the images efficiently, the proposed methods utilized the PACS system in the hospital. After sending the image data of each patient to a local monitoring computer, the patient's information, such as, name, age, male, and registration number were attached on the image. This file was then converted to a DICOM (digital imaging and communications in medicine) files and transferred to the PACS Server. The proposed system promises easy and convenient access on the images of lesions and various test results of patients to support fast and appropriate treatment.
\end{abstract}

Keywords: PACS; Real-Time Transmission; DICOM; Patient Examination; Wireless

\section{Introduction}

Due to the rapid development of information technology (IT), it is common for hospitals utilizing electronic medical record (EMR) and picture archiving and communication system (PACS) for obtaining, transferring and displaying patient data and images.

Images and various laboratory and other test results of patients are important information in diagnosing and treating the patients. Furthermore, some patients require continuous observation to monitor lesions or bedsores. For these patients, taking pictures of the site constantly and checking the status are essential parts of treatment. However, these photos are not managed well in general. Some images are simply attached in the patient's electronic chart but the quality of the image is not well enough and one cannot find the image later conveniently. The lack of storage management of the images could induce missing or delaying appropriate treatment for the patients.

It is difficult to establish a storage management system for the images in a general hospital since the system requires a lot of manpower and operating cost. In addition, training nurse and physicians is another problem of building the system. The system should be easy to use and convenient for this reason. This is why there is no such system that monitors the images of the lesions and test results of patients in real-time so far.

In this paper, we propose a storage management method that enables automatic transmission of the lesion and test image of the patients in a convenient manner. The proposed system can be easily attached to the conventional PACS system and does not require much cost for adopting the system. The system ensures real-time transfer of the images via wired or wireless network. There is no worry about the loss of the image.

\section{Materials and Methods}

\subsection{Diagram for Storage Management Methods}

The proposed scheme for storage management is illustrated in Figure 1.

When a nurse or a physician takes pictures of lesions or test results, the image is transferred to an automatic converting computer for converting the transferred images to DICOM (digital imaging and communications in medicine) format. After the conversion, files are uploaded to the PACS server. The uploaded images are now accessible from any computer in the hospital. The detailed process of the system will be described in the following sections. 


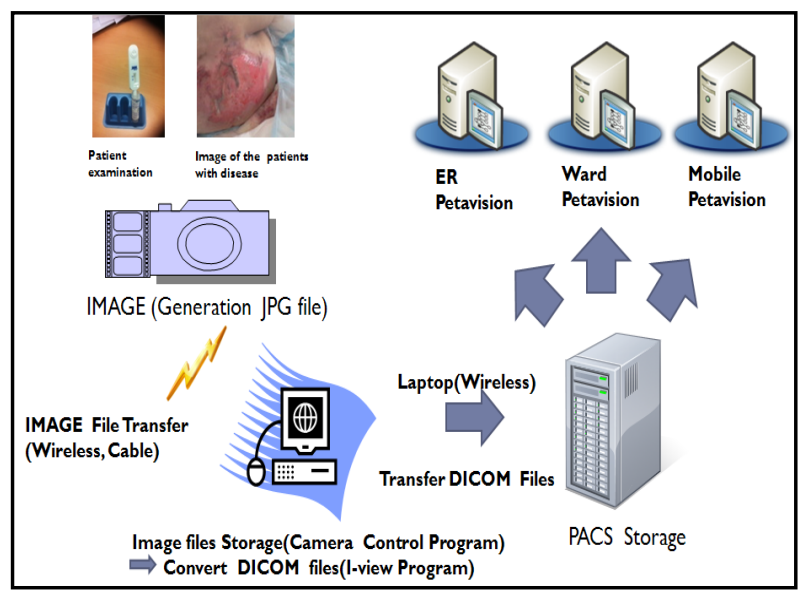

Figure 1. Schematic diagram of the proposed system.

\subsection{Picture Acquisition System}

To take pictures of lesions or test results of patients, a digital camera, which is connected to a laptop computer via a USB (universal serial bus) port or with wireless network [1], was used. The pictures were stored in a JPEG (joint photographic experts group) format. Figure 2 shows the camera unit (D60, Nikon, Japan) connected to a laptop computer (SENS R60, Samsung, Korea). Camera control program (camera control pro2, Ver. 2.13.0, Nikon, JAPAN) installed on the laptop automatically recognizes the camera device when the power is turned on and creates image files in JPEG format when the shutter on the camera pressed [2,3].

\subsection{Real-time Automatic Convert DICOM Files Transmission System}

The images that are stored in the patient's disease or testers need to match the patient information and physician's prescription. Patient information must be entered by the nurse to prescribe. To observe the patient's disease continuously, we build a database of information and images of the patient. Figure 3 shows the workstation laptop computer installed the real-time automatic convert DICOM file transmission program (iview, Ver. 2.0.00, CAD Impact. Inc, KOREA).

After entering patient information in accordance with the prescribed is stored should be matched with the images in a specific folder. The real-time automatic convert DICOM file transmission program automatically convert to DICOM file $[4,5]$. The DICOM file transfer PACS Storage to designated IP address. The laptop computer using the Wireless LAN card (ATHEROS, 802.11b.g) is sent to the PACS wireless.

\subsection{Real-Time PACS System}

Figure 4 shows The PACS System that is installed in the

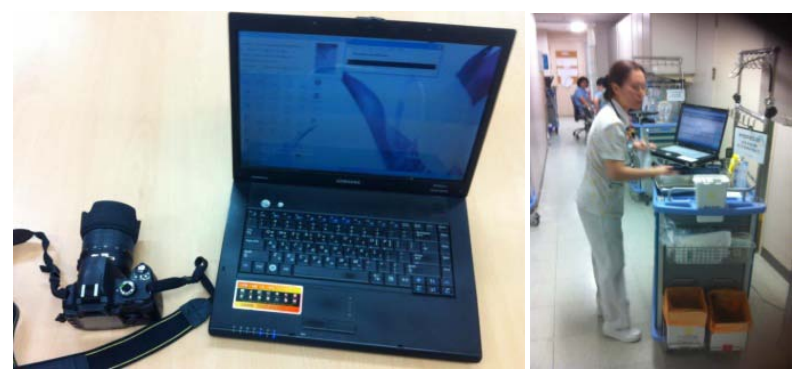

Figure 2. Camera unit and laptop computer.

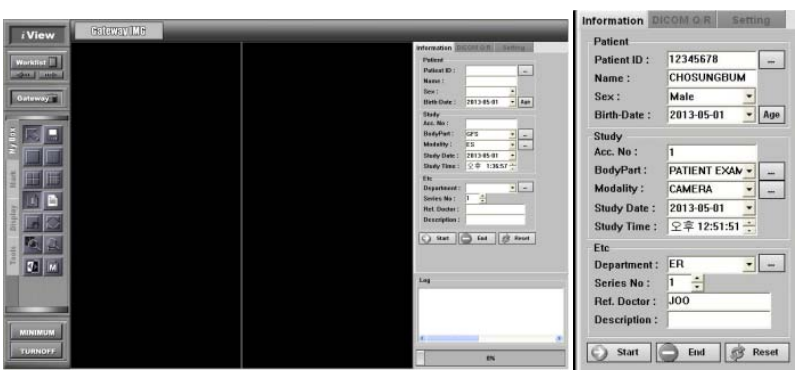

Figure 3. Program for real-time conversion to DICOM file and automatic transmission.

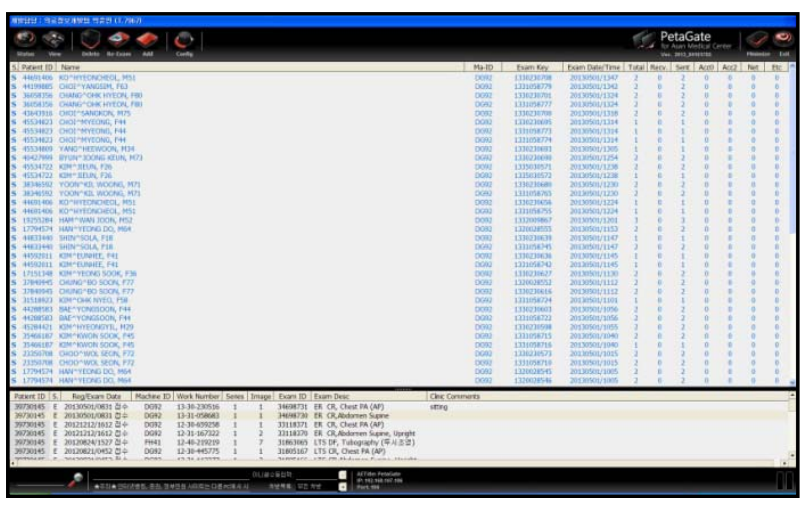

Figure 4. PACS gateway (PETAGATE program).

hospital. The system consists of the PACS gateway, PETAGATE program (PETAGATE, Ver. 201305021655, and SEOUL ASAN Medical Center, KOREA) and PACS Viewer program, PETAVISION program (PETAVISION, Ver. 2.1.0.1012, Asan Medical Center, KOREA).

The PACS gateway, PETAGATE program before it is sent to the PACS server DICOM file to final inspection. DICOM PACS gateway permits to connect the PACS Server. The PACS Viewer, PETAVISION program is an easy-to-use application that allows users to view medical DICOM images made from PACS Storage.

\section{Result and Discussion}

Storage management methods for automatic image transmission of the image of the patients with disease and patient examination were designed and initiated in 2013 for continuous observation and treatment Service. 


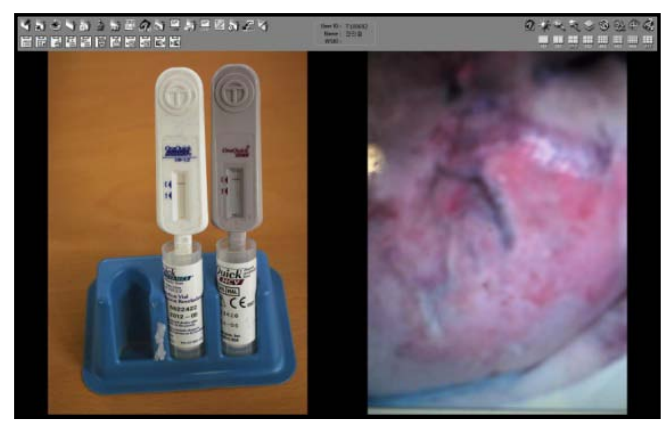

Figure 5. PACS viewer (PETAVISION program).

After Patient information is entered to program information window in workstation laptop computer, as soon as images of the patients with disease and examination are generated, they are automatically transferred to workstation laptop computer and convert to DICOM file. Whenever DICOM file are generated, it is automatically transfer to PACS gateway real-time wireless. Physician, nurse, particular administrator of patient disease could check the information of patient image from anywhere in the hospital PACS System.

Because it is already using a laptop computer equipped the Wireless LAN card in the ward for EMR, Mobile Workstation Laptop Computer-based environment was implemented to reduce cost and take advantage of userfriendliness, time, satisfaction of the user. Tremendous benefits of Mobile Wireless Laptop Computer-based environment see through PACS and Anywhere easily store image of the patient with diseases and patient examination. In addition, it is only the cost of DICOM convert program, Camera Control program and Camera Unit.

Seoul Asian Medical Center PACS was designed with its own technology and initiated in 2000. All radiologic imaging including outpatient radiography are supported by the PACS. In addition, Mobile HIS (Hospital information system) has been built in 2010 became available mobile-OCS/EMR/PACS. We can anytime, anywhere check the patient information of the emergency room, hospitalization, wards, in the operating room.

Images with the disease or the patient tester are important information for observation and treatment of patients with. Information and data of such patients should not to be neglected, it should be managed efficiently.

To keep a fast speed and good quality, hospital must be upgraded or replace laptop computer. If laptop computers are replacing with the latest mobile PC, Physician and nurse can explain Treatment over image to the patient through a separate monitor. So, Storage manage- ment methods for improving the image of the patients and patient examination at the hospital are currently being tested continuously.

\section{Conclusions}

In this study, we presented an improved management system for managing patient pictures of specific lesion or various test results. The system transfers the acquired image of patients to a PACS system in a hospital. The image data of each patient contains the patient's information, such as, name, age, male, and registration number since the file is converted to a DICOM format before transferred to the PACS Server. The proposed system promises easy and convenient access on the images of lesions and various test results of patients to support fast and appropriate treatment.

Real-time transfer of the images via network allowed medical doctors to monitor the status of the patient's diseases, or examination would undergo continuous observation and treatment. It was also able to explain the progress of patients with diseases using the mobile pc or mobile phone connected to the PACS.

The wireless data transmission could provide better data management, reduced data transfer time, and convenient operation.

Sensitive information, such as images of the disease, or patient tester will be stored and managed on the PACS. As a result, Nurse Representative is not discomforted, and satisfaction increases.

\section{Acknowledgements}

This research was supported by Basic Science Research Program through the National Research Foundation of Korea (NRF) funded by the Ministry of Education, Science and Technology (2010-0007281).

\section{REFERENCES}

[1] Radio-Frequency Wireless Technology in Medical Devices DRAFT GUIDANCE, FDA, USA, 2007.01

[2] "The Implementation of Homecare Nursing Network System Using Wireless Network," The Korean Society of Medical Informatics, Vol. 7, No. 1, 2001, pp. 13-21.

[3] D60 Digital Camera Operation Manual, CANON, 2008

[4] Digital Imaging and Communications in Medicine (DICOM), National Electrical Manufactures Association, 2001.

[5] “iView User’s Guide,” CAD Impact. Inc, 2010 